Final optics damage inspection (FODI) for the National Ignition Facility

A. Conder, T. Alger, S. Azevedo, J. Chang, S. Glenn, L. Kegelmeyer, J. Liebman, M. Spaeth, P. Whitman

November 12, 2007

Boulder Damage Symposium

Boulder, CO, United States

September 24, 2007 through September 26, 2007 
This document was prepared as an account of work sponsored by an agency of the United States government. Neither the United States government nor Lawrence Livermore National Security, LLC, nor any of their employees makes any warranty, expressed or implied, or assumes any legal liability or responsibility for the accuracy, completeness, or usefulness of any information, apparatus, product, or process disclosed, or represents that its use would not infringe privately owned rights. Reference herein to any specific commercial product, process, or service by trade name, trademark, manufacturer, or otherwise does not necessarily constitute or imply its endorsement, recommendation, or favoring by the United States government or Lawrence Livermore National Security, LLC. The views and opinions of authors expressed herein do not necessarily state or reflect those of the United States government or Lawrence Livermore National Security, LLC, and shall not be used for advertising or product endorsement purposes. 


\title{
Final Optics Damage Inspection (FODI) for the National Ignition Facility
}

\author{
Alan Conder, Terry Alger, Stephen Azevedo, Jim Chang, Steven Glenn, Laura \\ Kegelmeyer, Judith Liebman, Mary Spaeth, Pam Whitman \\ University of California \\ Lawrence Livermore National Laboratory \\ 7000 East Avenue, P. O. Box 808, L-463 \\ Livermore, CA 94551 \\ Phone: 925-422-2032 \\ E-mail: conder1@,llnl.gov
}

Keywords: FODI, NIF, optics inspection, laser-induced damage, high-resolution image analysis

\begin{abstract}
The National Ignition Facility (NIF) at the Lawrence Livermore National Laboratory (LLNL) will routinely fire high energy shots (approaching $10 \mathrm{~kJ}$ per beamline) through the final optics, located on the target chamber. After a high fluence shot, exceeding $4 \mathrm{~J} / \mathrm{cm}^{2}$ at $351 \mathrm{~nm}$ wavelength, the final optics will be inspected for laser-induced damage. The FODI (Final Optics Damage Inspection) system has been developed for this purpose, with requirements to detect laser-induced damage initiation and to track and size it's the growth to the point at which the optic is removed and the site mitigated. The FODI system is the "corner stone" of the NIF optic recycle strategy. We will describe the FODI system and discuss the challenges to make optics inspection a routine part of NIF operations.
\end{abstract}

This work performed under the auspices of the U.S. Department of Energy by Lawrence Livermore National Laboratory under Contract DE-AC52-07NA27344.

\section{Overview}

The National Ignition Facility (NIF) will achieve fusion ignition using the focused energy from 192 high power lasers to compress and heat a fuel capsule (target) positioned at the center of the NIF target chamber. The NIF is designed to deliver 1.8 million joules of ultraviolet laser energy and 500 terawatts of power on target.

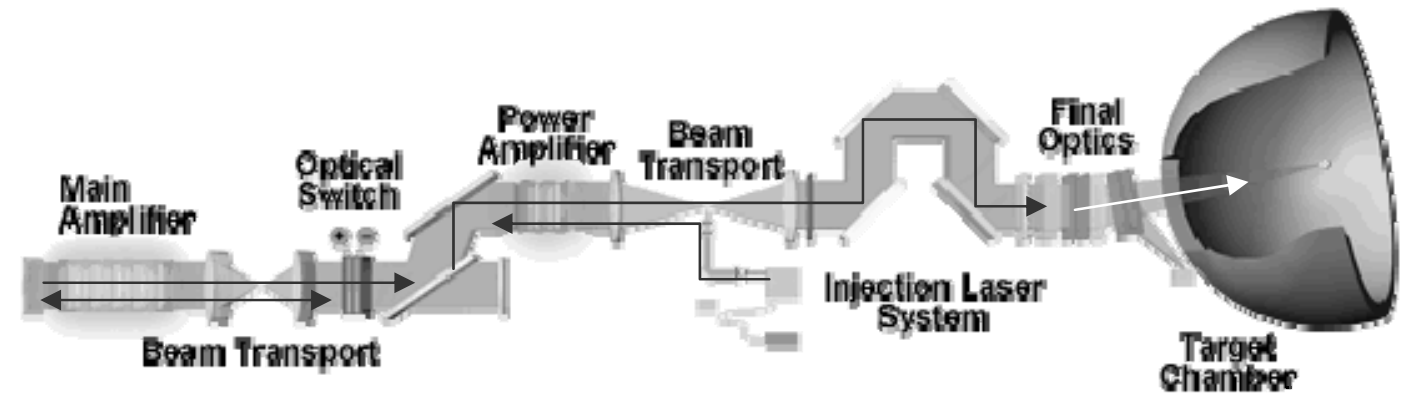

Figure 1 Schematic of a single beamline on NIF. A low energy laser pulse is injected into the beamline and makes several passes through the amplifiers before the optical switch opens and allows the resulting high energy laser pulse to proceed to the target chamber. 
Most large aperture optics within the NIF beamline will be exposed to a high energy laser pulse while its photons have a wavelength of $1.053 \mu \mathrm{m}(1 \omega)$. At this wavelength, the laser-induced damage threshold as a function of fluence $\left(\mathrm{J} / \mathrm{cm}^{2}\right)$ is sufficiently high to enable operation of the NIF with minimal risk to the optic surfaces.

In the final optic section of the laser, located at the NIF target chamber, nonlinear frequency conversion crystals convert the high energy laser pulse to the ultraviolet wavelength of $351 \mathrm{~nm}$ $(3 \omega)$. The final focusing lens focuses the $3 \omega$ pulse onto the target in the target chamber, where the one hundred ninety two beamlines converge and drive the target to fusion ignition.

At the $3 \omega$ wavelength, the laser-induced damage threshold of the optics is lowered, putting the optics in the $3 \omega$ section of the laser at higher risk to laser-induced damage. These optics may be operated near their damage threshold for the NIF to deliver on its primary mission - fusion ignition.

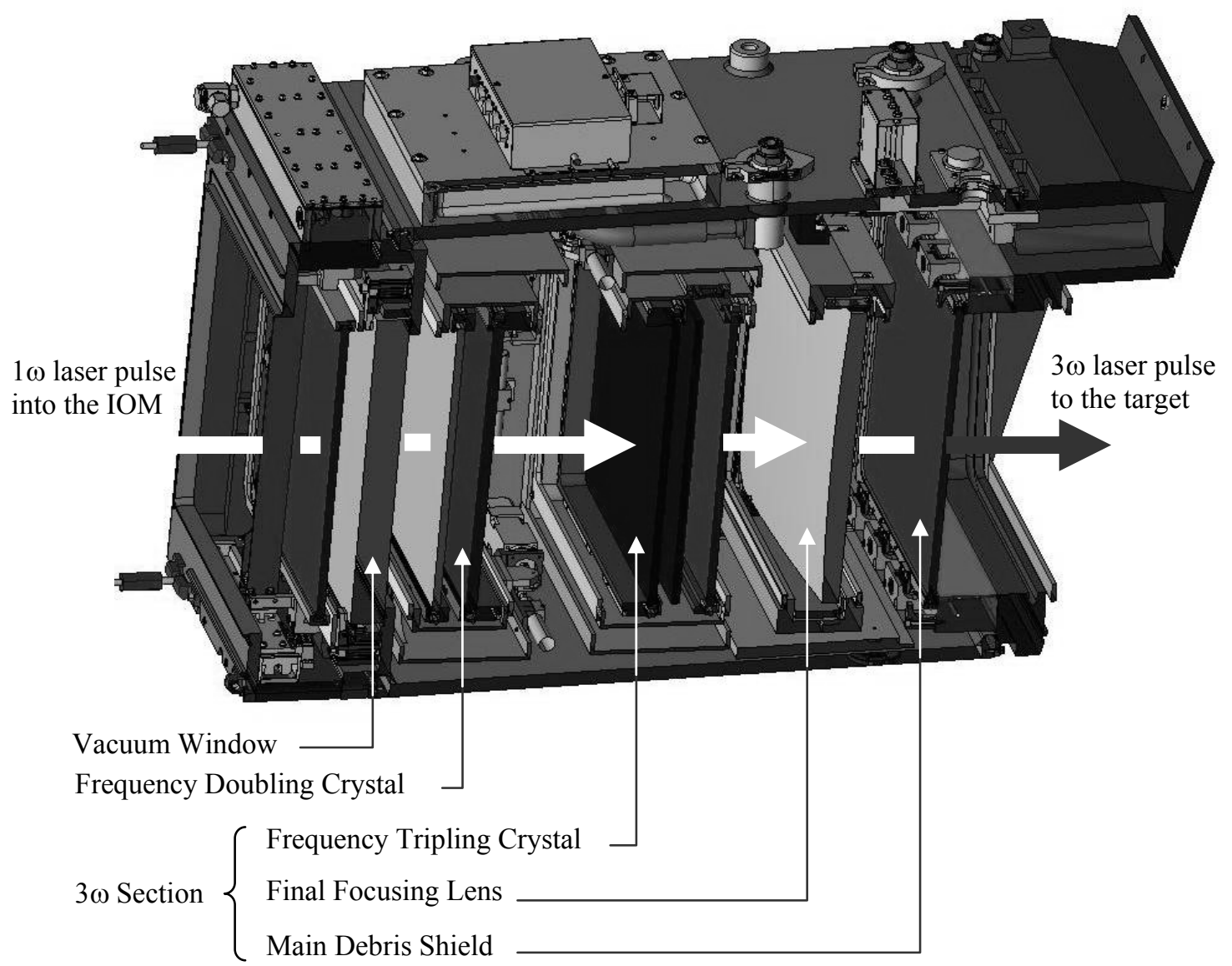

Figure 2 Integrated Optics Module (IOM) houses the final optics, including those exposed to the 3 high energy laser pulse. These include the nonlinear frequency conversion crystals the fused silica final focus lens and the main debris shield

When the $3 \omega$ fluence exceeds $4 \mathrm{~J} / \mathrm{cm}^{2}$ there is a finite probability that a damage site will initiate on the surface of the optic. The size of the initiated site is very small, less than $50 \mu \mathrm{m}$. For the fused silica final focus lens and the main debris shield, this site may continue to grow on 
subsequent laser shots at a rate that is dependent on the total fluence of each shot. The Final Optics Damage Inspection (FODI) system is designed to image the optics in the IOM and detect damage initiation and track growth. When the site has grown to the maximum allowed size, the optic will be removed and sent to the NIF damage mitigation facility, where the site will be mitigated and the optic repaired. The optic will then be returned to the NIF inventory.

\section{Final Optics Damage Inspection (FODI) System}

The Final Optics Damage Inspection (FODI) system is an optical telescope designed to be inserted to the center of the NIF target chamber after each laser shot. From this position it can point into each beamline and acquire images of the final optics within the IOM. These images are analyzed by the optics inspection analysis software that detects flaws on the surface of an optic that appear in the FODI image.

The FODI instrument is controlled by the NIF Integrated Command and Control Software (ICCS). This software positions the instrument to point to each beamline using predetermined set points and to focus and acquire image sets of the required optics.

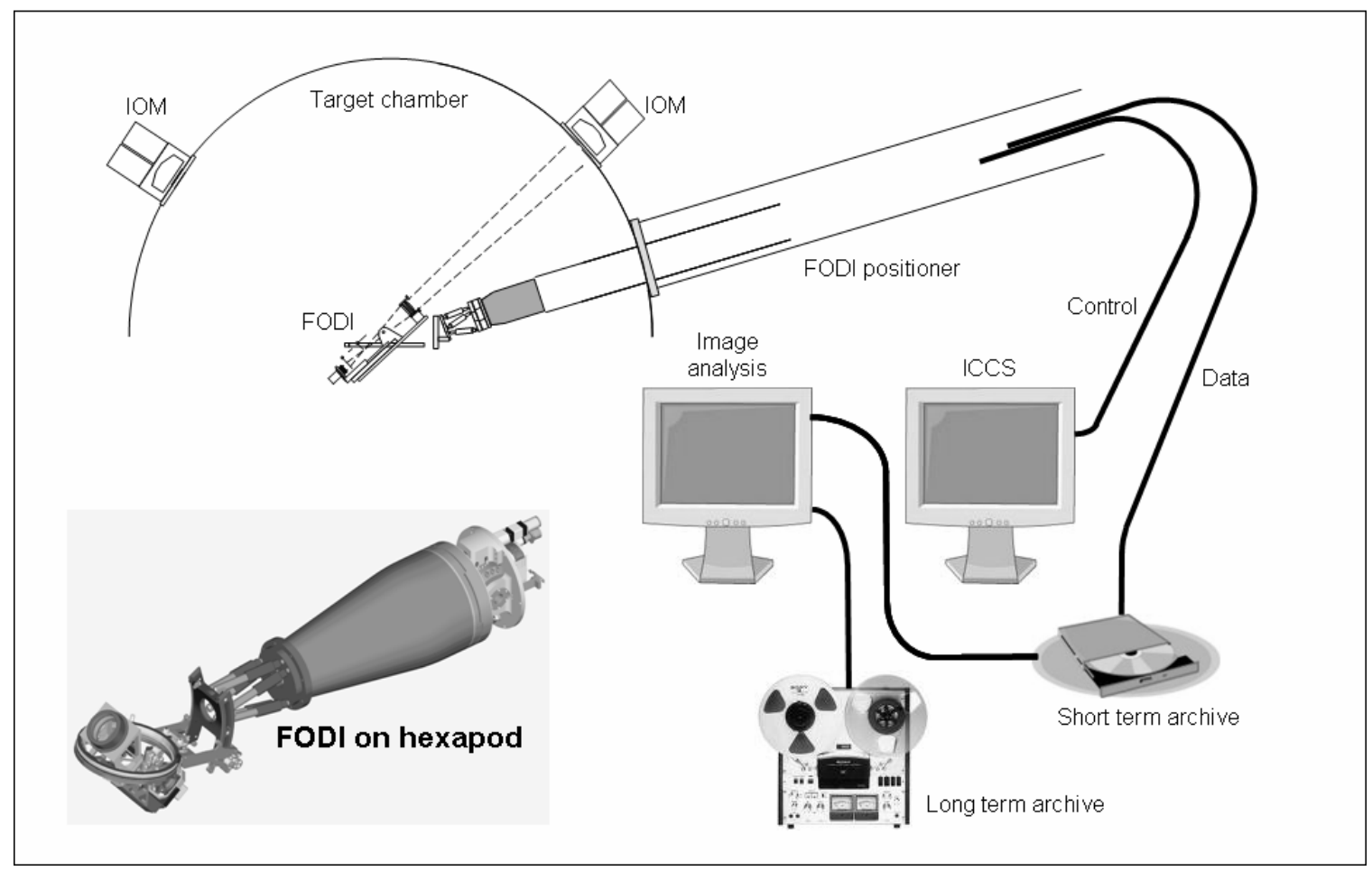

Figure 3 FODI is an integrated inspection system involving a positioner to move FODI to the center of the target chamber, a telescope capable of pointing into each beamline using a motorized mount, control and image acquisition software, and image analysis tools used to search the acquired images for flaws on the surface of the imaged optics 


\section{FODI System Requirements}

The FODI instrument and its optics inspections algorithms are required to detect laser-induced damage after it initiates on the surface of an optic; when the diameter of the site is as small as 50 $\mu \mathrm{m}$. FODI is required to track each site and determine its size after each inspection. When the size of the site approaches an allowable size, currently $300 \mu \mathrm{m}$ for the focus lens, the optic can be removed and the site mitigated; the optic is then returned to inventory for future use.

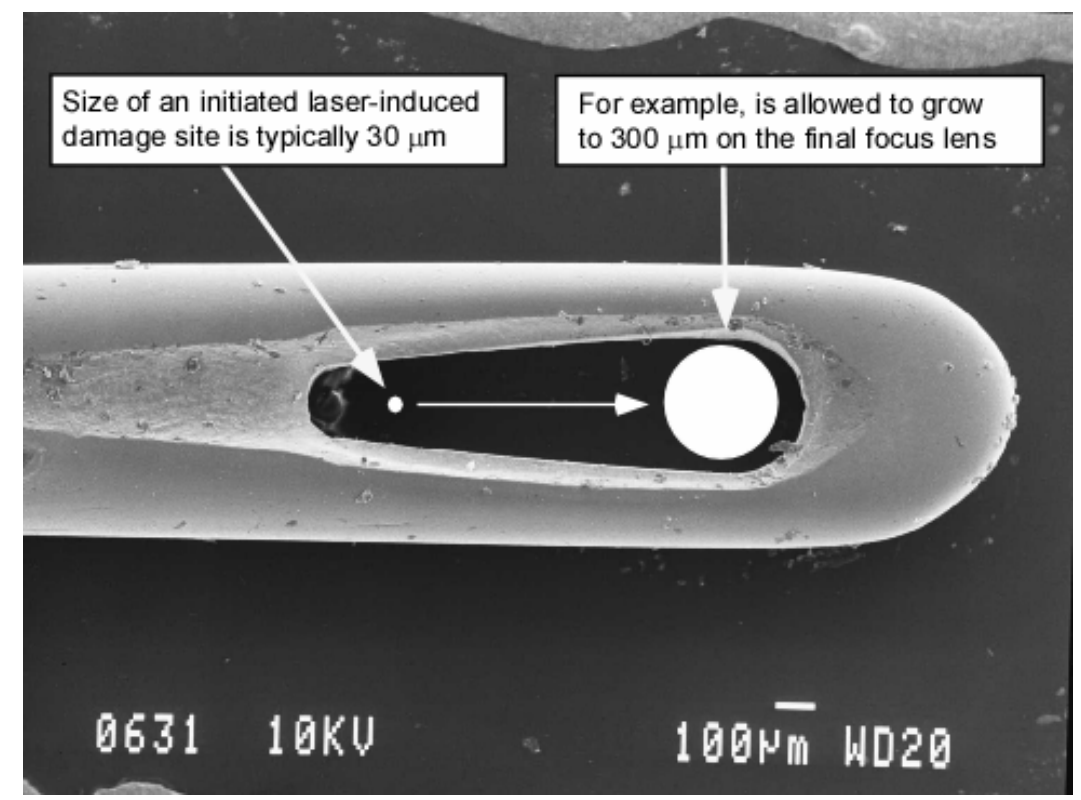

Figure 4 Electron microscope image of the eye of a needle revealing the size that laser-induced damage may initiate on the final focus lens and the allowable size to which it may grow before the optic is removed and repaired.

The following list of requirements for the FODI system reveals the challenge involved in designing this system for operational use in the NIF:

- Detect sites at sizes of $\geq 50 \mu \mathrm{m}$ with $99 \%$ confidence ( $3 \omega$ optics)

- Calculate the size of the detected sites with an accuracy of $1 \sigma \leq \pm 15 \%$

- Provide a history of the size of each site to track growth over time

- Provide accurate $\mathrm{x}, \mathrm{y}$ coordinates (in physical units $\mathrm{mm}$ ) of each site on the optic

- For 192 beams, within two hours

- From the center of the target chamber, at a distance of more than seven meters from the optic being imaged, and while under vacuum at $<10^{-4}$ Torr 


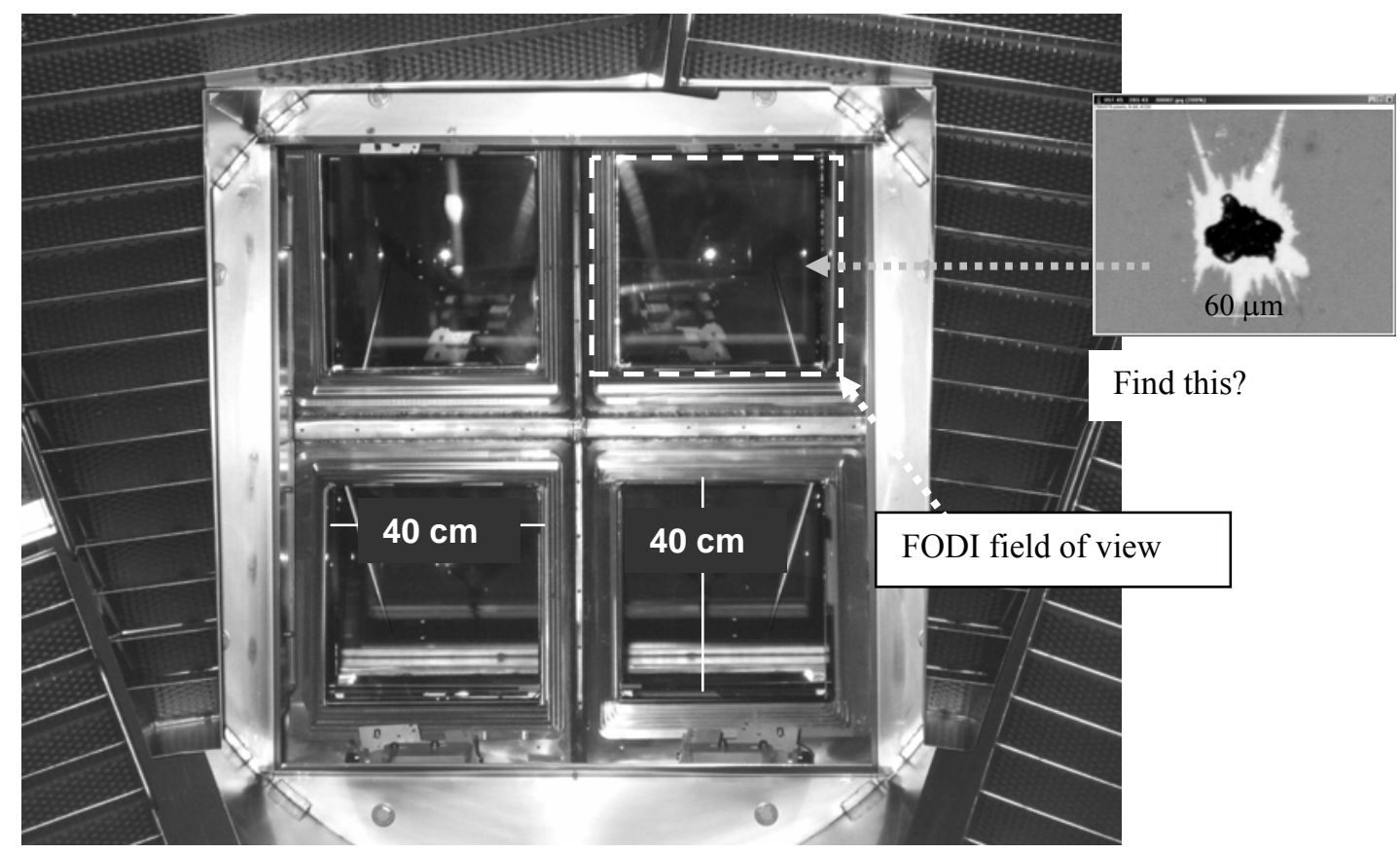

Figure 5 View of the IOM outputs of four beamlines as seen from the center of the target chamber. FODI will point into each beamline and acquire images of each optic in the IOM

The small size of flaws and damage sites to be detected and sized by FODI, as seen from the center of the target chamber, and relative to the total surface area of the optics to be inspected, is equivalent to...locating a contact lens floating on the surface of a pond, $1 / 2$ mile diameter, from an altitude of 1600 feet!

\section{FODI Instrument Description}

The FODI instrument is attached to the end of a Diagnostic Instrument Manipulator (DIM) that inserts FODI close to the center of the target chamber, where the FODI hexapod, a six degree of freedom manipulator, precisely aligns FODI to the target chamber coordinates using predefined setpoints. The FODI telescope can then point into each beamline using motorized azimuth, elevation, and roll stages and predefined setpoints for each beamline position.

A high resolution CCD camera, mounted on the FODI telescope, is focused to each optic position using a linear translation stage and predefined focus setpoint and acquires an image of the optic. When the image acquisition of the optic is complete the camera moves to the next optic position. This sequence continues until images have been collected of each optic in the IOM. At this point the FODI instrument can be pointed to the next beamline and the image acquisition sequence repeats. 

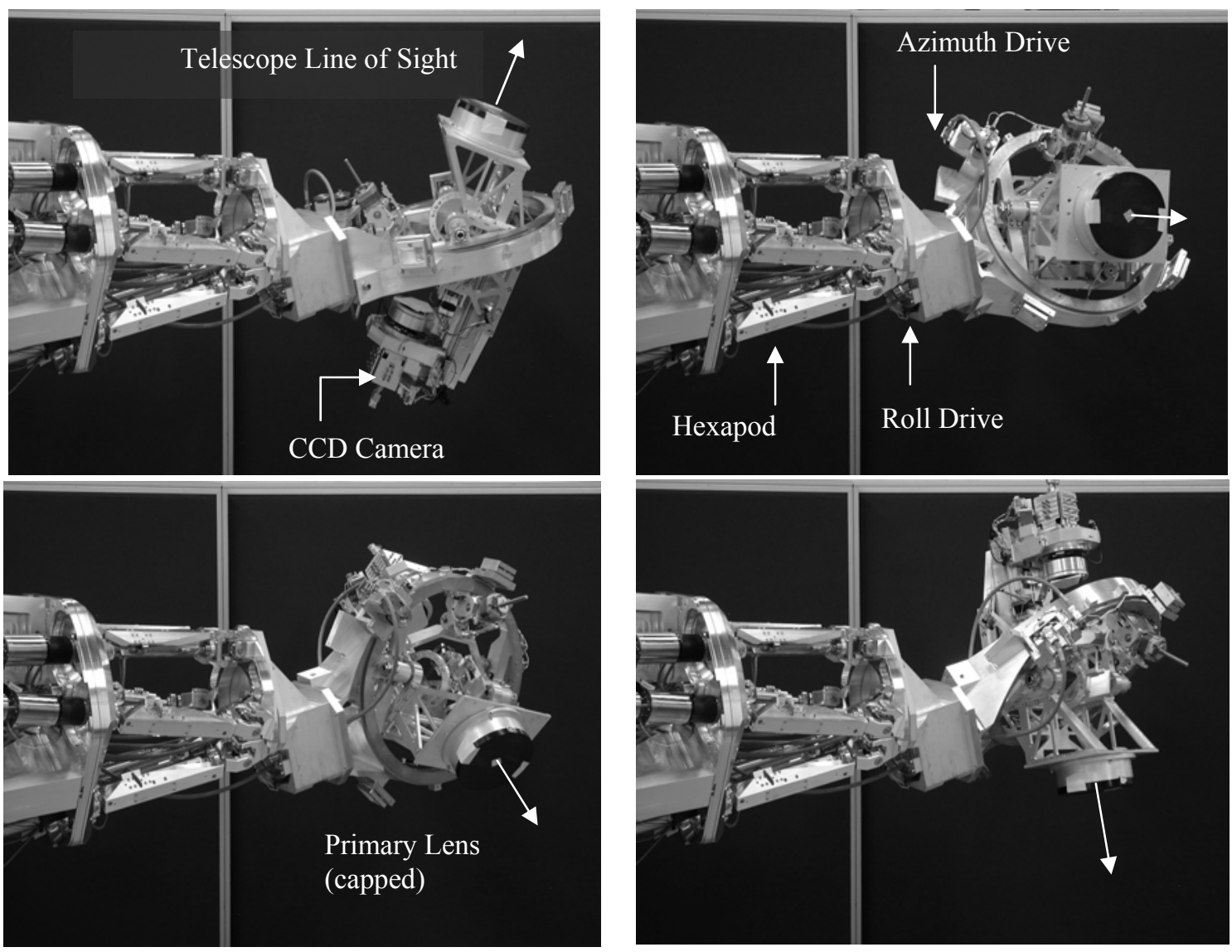

Figure 6 FODI telescope is capable of pointing into any beamline on the target chamber using motorized azimuth, elevation and roll stages. The hexapod is a six degree of freedom manipulator used to align FODI to the target chamber coordinates

\section{FODI telescope specifications:}

- $\quad$ Azimuth Range, Resolution: $\quad 357^{\circ}, 0.001^{\circ}$

- $\quad$ Elevation Range, Resolution: $20^{\circ}$ to $77^{\circ}, 0.001^{\circ}$

- $\quad$ Roll Range, Resolution: $\quad 270^{\circ}, 0.001^{\circ}$

- $\quad$ Hexapod Range at TCC: $\quad \pm 30 \mathrm{~mm}(\mathrm{x}, \mathrm{y}, \mathrm{z})$ and $\pm 3^{\circ}(\Phi \mathrm{x}, \Phi \mathrm{y}, \Phi \mathrm{z})$

- Hexapod Resolution at TCC: $\quad \pm 30 \mu \mathrm{m}(\mathrm{x}, \mathrm{y}, \mathrm{z})$ and $\pm 0.001^{\circ}(\Phi \mathrm{x}, \Phi y, \Phi z)$

- $\quad$ Working Distance: 5 meters to 80 meters

- $\quad$ Telescope f/number Range: $\mathrm{f} / 4$ to $\mathrm{f} / 160$

- $\quad$ Telescope Focal Length: $610 \mathrm{~mm}$

- $\quad$ CCD Image Format: 4,000 x 4,000 pixels @ 14-bits

- Pixel FOV: $\quad 110 \mu \mathrm{m}$ at 8 meters working distance 
The f/number for the FODI imaging system generates a relative shallow depth of field, $\sim 10 \mathrm{~mm}$. This creates a problem when imaging the transport mirrors in the NIF beamline, upstream of the IOM, in the $1 \omega$ section of the laser. FODI is required to inspect the surfaces of these mirrors for the unlikely event that laser-induced damage occurs. All transport mirrors have surfaces that are tilted with respect to the FODI line of sight, in some cases by $45^{\circ}$. This results in a variation of focus across the FODI field of view, causing a blurring of objects on the optic surface as they move from best focus, increasing loss of peak signal that can adversely affect the detectability of small features in the image.

To overcome this problem FODI incorporates the Schiempflug technique into its design. This technique requires tilting the CCD image plane opposite and proportional to the tilt of the object plane resulting in an in-focus image across the entire optic surface. To achieve the Schiempflug condition the FODI CCD camera is mounted to a two axis gimbal.

A significant constraint on the FODI instrument design is the need for FODI to operate for several hours in vacuum. This requires attention to the thermal management of the active components in the instrument, such as the CCD camera, power supply, and other electronics. A water coolant loop is designed into the system to remove the exhaust heat from the electronics and pass it to a heat exchanger external to the target chamber. In addition, materials used to construct FODI have all been reviewed and tested for compatibility with the NIF target chamber vacuum environment.

\section{Optic Illumination Techniques}

Proper illumination of the optic to be imaged is critical for the successful performance of the FODI system. Three methods will be employed to illuminate the optics, bright-field back illumination, dark-field back illumination and edge illumination. Back illumination uses the NIF full aperture alignment beam to backlight the optic and is available for all the optics in the NIF beamline.
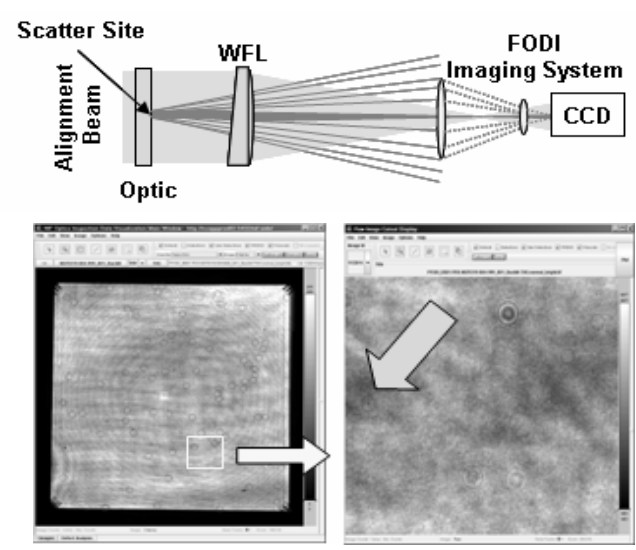

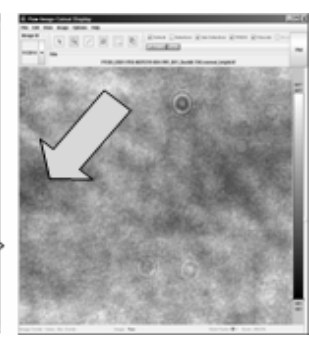

(a)

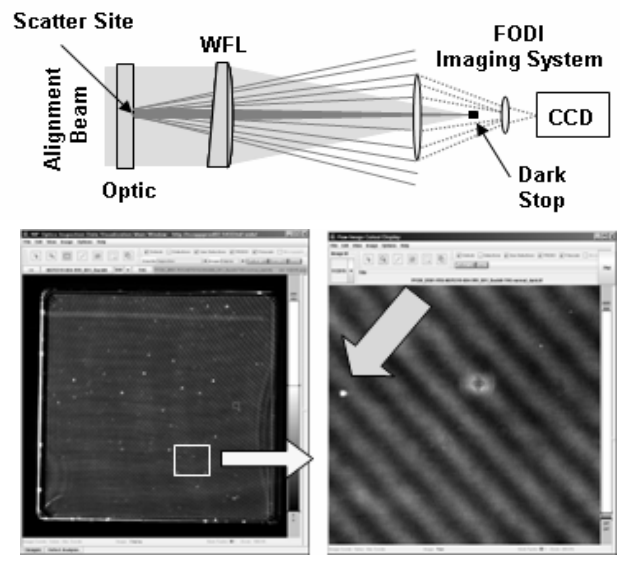

(b)

Figure 7 Images acquired by FODI (a) Bright-field backlit image, a 100 $\mathbf{m}$ flaw is difficult to detect, (b) dark-field backlit image reveals the same flaw with some background clutter 
When an optic is backlit, the light travels through the optic; a flaw on its surface will scatter the incident light, creating a dark silhouette against a bright background. This is known as brightfield imaging. This technique is adequate for locating large flaws on optics and identifying phase objects that are otherwise transparent. A phase object will tend to focus the incident light, and can appear brighter than the background. The performance of the FODI system using brightfield illumination is limited because of the high noise in the background that often obscures small objects. Therefore, bright-field illumination will not be relied upon to detect and size small flaws on the optics within the IOM.

As a method for increasing signal to noise, the FODI instrument can operate in dark-field mode by inserting a motorized stop at the far field focus in the imaging system. In dark-field imaging the bright background is blocked, while the high angle light scattered from the flaw on the optic surface passes around the stop and is imaged by the camera. The flaw will now appear bright against a dark background. Dark-field imaging enables detection of small flaws; however when two or more optics are in close proximity it can be difficult to discriminate on which optic the flaw occurs.
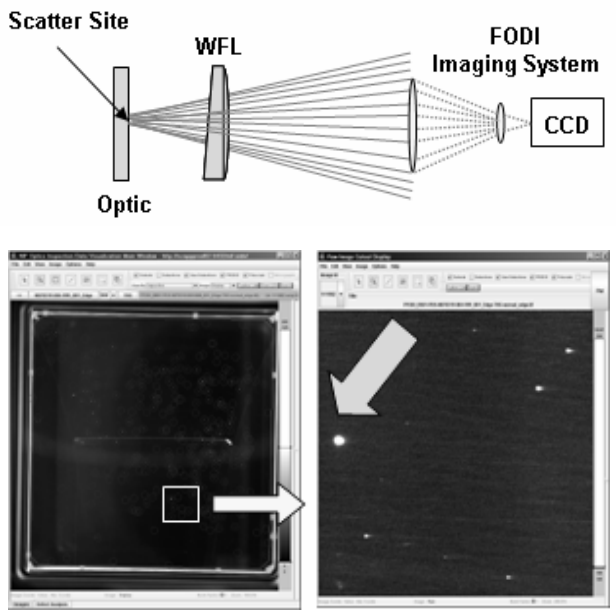

Figure 8 Edge illumination lights only the optic to be imaged and provides excellent signal to noise performance. The $100 \mu \mathrm{m}$ flaw is readily apparent in the edge lit image along with several smaller sites not seen in either back illuminated images.

Edge illumination is also used for several optics in the IOM: the frequency crystals, final focus lens, and the main debris shield. This method relies on light injected at the proper angle into the edge of the optics that subsequently becomes trapped through total internal reflection (TIR). A flaw on the optic surface disrupts the TIR and causes light to scatter from the optic at the flaw location. A percentage of this scattered light is collected and imaged by the FODI optical system, resulting in an image of the flaw, which appears bright against a dark background; and without the uncertainty of which optic the flaw belongs.

The edge illumination system was developed specifically for the NIF final optics. Fiber optics routed to the IOM carry light from a high-power laser diode located in a remote equipment rack. Light from the laser diode is launched into the fiber network through a 1:N fiber switch that is controlled by the software that controls the image acquisition of the FODI instrument. This switch diverts light from the laser diode to the fiber in the network that is coupled to the optic to be imaged. 


\section{Optics Inspection Analysis}

Each image acquired by the FODI instrument generates a file size of $34 \mathrm{MB}$. Typical inspections across 192 beamlines may produce 960 images, representing more than 33 GB of data per inspection. It is therefore important to use automated image analysis to process each image quickly and identify candidate sites on each optic that may correspond to laser-induced damage.

Optics inspection (OI) algorithms, developed for NIF optics inspection, read each FODI image and execute algorithms to search for and detect physical sites in the imagery, while rejecting background noise in the image that appears in the forms of ghosts and reflections. Once all possible detections are made, the OI algorithms further refine the analysis to provide a confidence metric on the likelihood that detections represents a true physical feature on the optic, resulting in high confidence detections that correlate to flaws and damage on the surface of the optic.

An important function of the OI analysis is to locate the physical coordinate of each site on the optic surface. To facilitate this process, fiducials are placed in a known position on the optic and are detected using the OI algorithms. Using pattern recognition codes to identify each fiducial pattern, the OI algorithms can use the fiducial locations in the image to convert image coordinates of each detected site into physical coordinates on the optic.

With the optic serial number and the physical location of the site, all high-confidence detections are uniquely identified and their history tracked. This provides valuable information on the growth of each damage site and allows off-line facilities to quickly locate and repair the damage site on the optic.

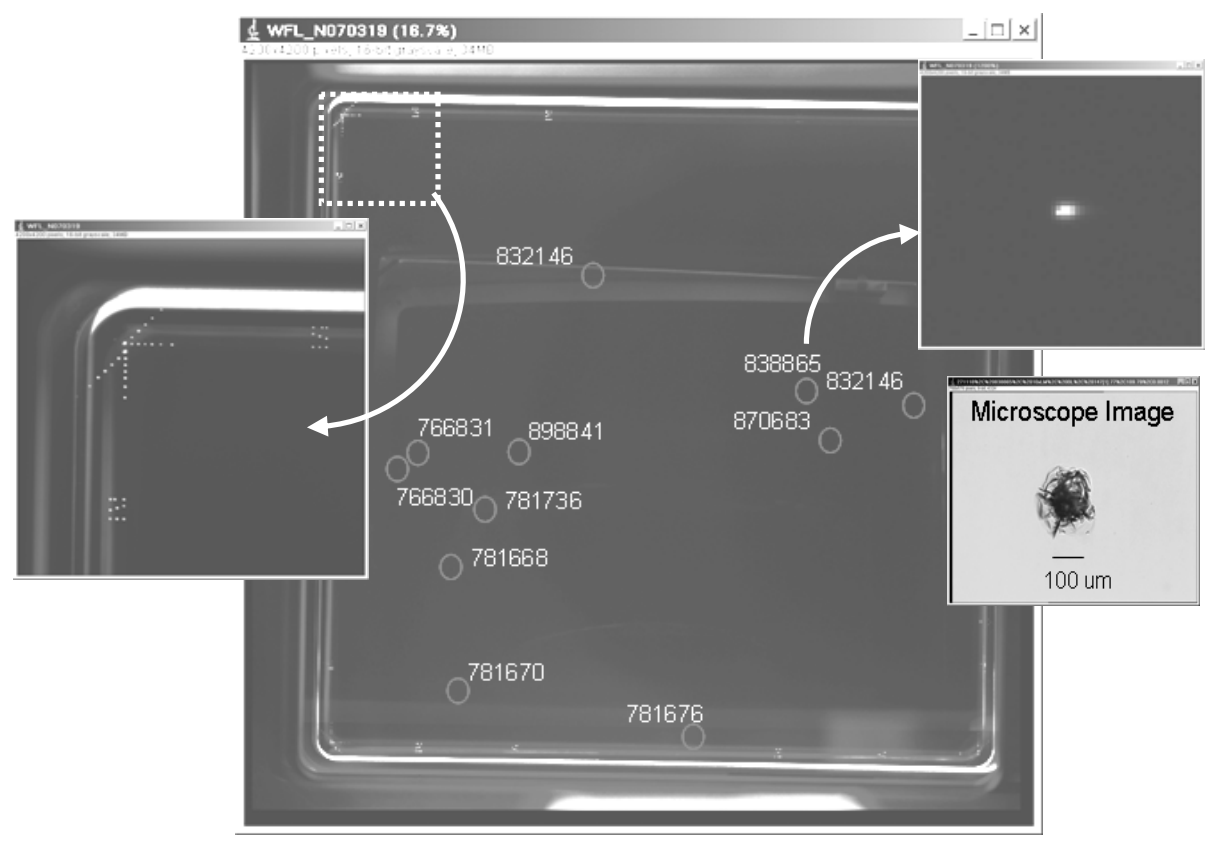

Figure 9 An edge lit image of the final focus lens showing the high confidence detections circled in red and identified. Note the image of the crystal carrier in the background. The optics inspection algorithms are able to locate even the smallest sites in the presence of this background clutter. 


\section{Defect Sizing Techniques}

The size of a FODI camera pixel scaled to an optic inside the IOM is approximately $110 \mu \mathrm{m}$. It is not possible therefore to resolve a flaw on the surface of the optic with a diameter of less than $300 \mu \mathrm{m}$. Instead, FODI relies on radiometry; using the integrated signal captured from a laserinduced damage site to calculate the area and size of small sites. Successful use of radiometry requires calibration, accomplished by installing on a beamline, at each edge lit optic position, an optic with a range of known and measured damage sites. From this truth data, a calibration equation is generated for the specific optic type, and is used by the OI analysis software to convert total integrated signal into an estimated diameter.

Fiducials play an important role in maintaining this calibration. Their total collected signal is measured during calibration and tracked. The calibration can be scaled relative to the detected signal from the fiducials to account for changes in system sensitivity. The results of using the radiometric method for sizing laser-induced damage are very promising and indicate that FODI can detect and accurately size damage sites from $30 \mu \mathrm{m}$ to several millimeters; providing a dynamic range of 100:1.

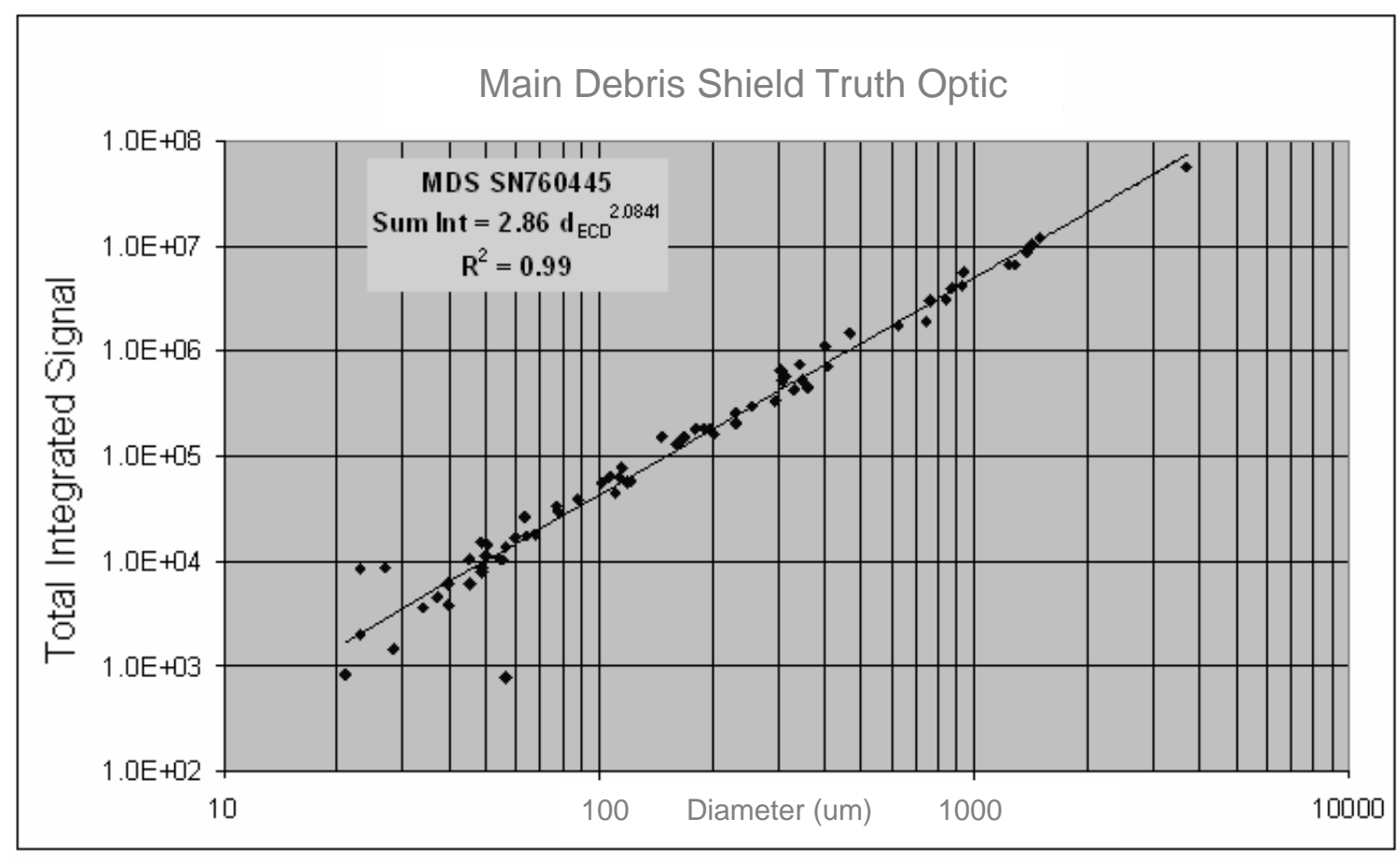

Figure 10 Calibration curve generated from a main debris shield truth optic that had damage sites that were allowed to grow large to validate the dynamic range of the radiometric method of sizing damage sites with FODI. 


\section{Conclusions}

Moving the FODI instrument and DIM from the production facility to the NIF building was an engineering feat requiring the effort of many dedicated individuals. The DIM alone weighs nearly 18,000 lbs and required great attention to safety during the move. Initial testing of the FODI telescope design has shown that FODI can meet its strict performance requirements and expert tools have been developed and are operational for automated analysis of the FODI imagery, resulting in efficient inspection of the NIF final optics.

The FODI system is installed in the NIF building and is currently being commissioned for operations beginning in the Fall of 2007.

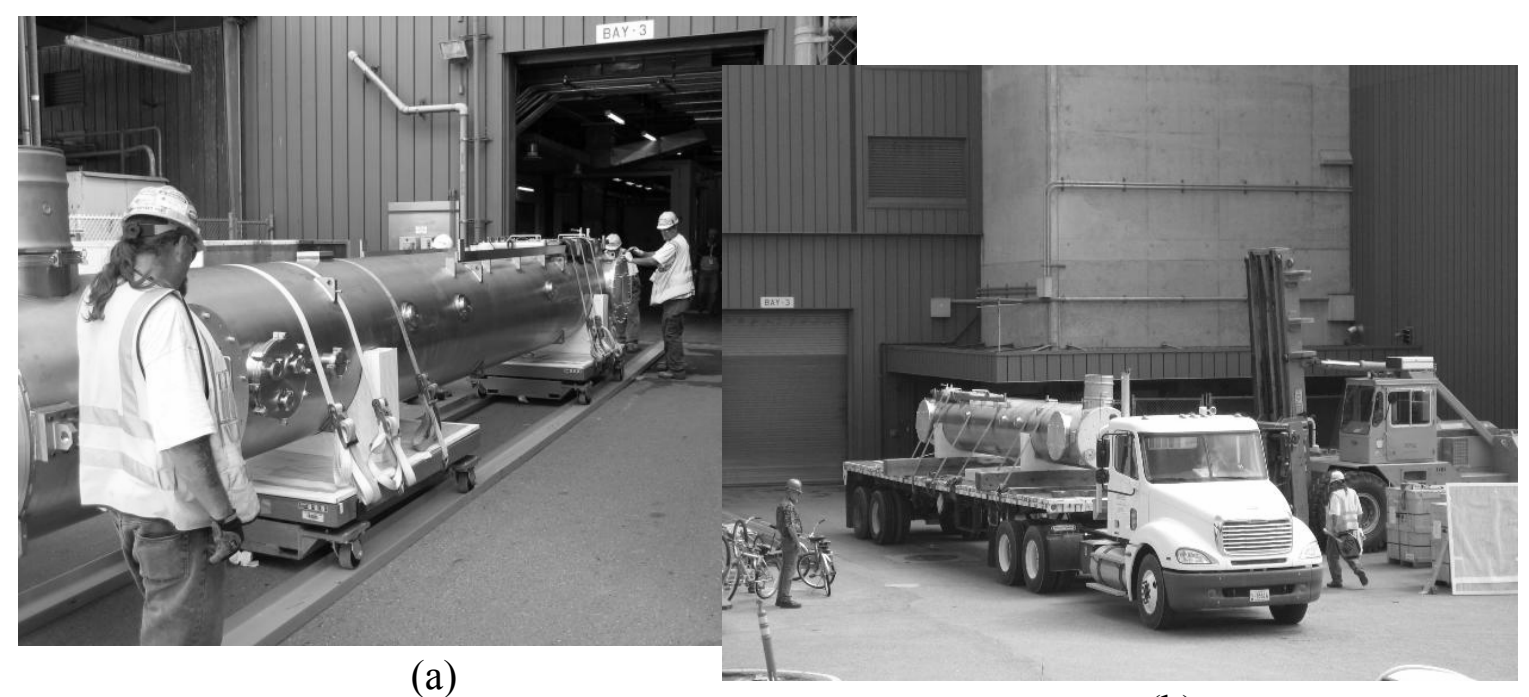

(b)

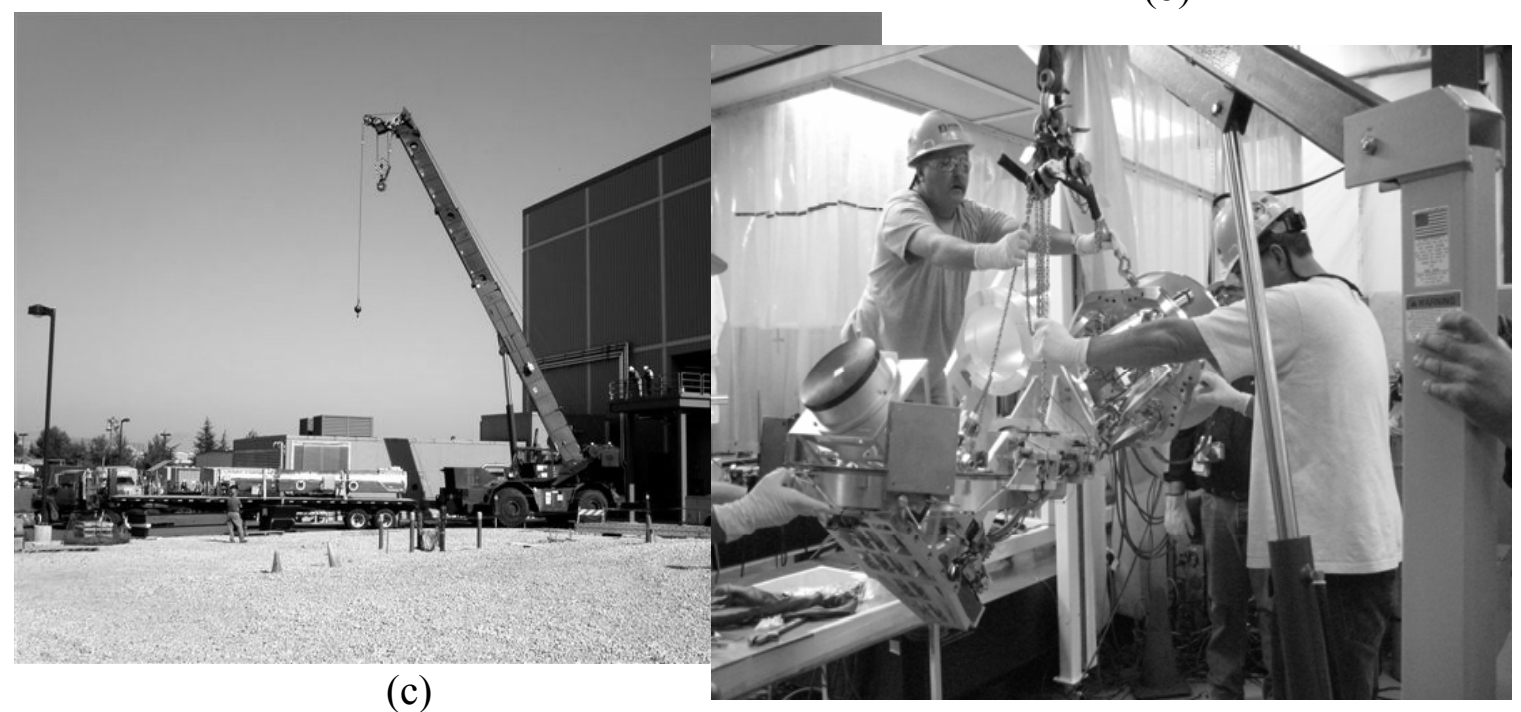

(d)

Figure 11 (a) Moving the FODI DIM from the production facility; (b) loading it onto an air ride flat bed, note the large fork lift; (c) preparing for a crane lift it into the NIF; (d) and the FODI instrument being hoisted in preparation for its move to the NIF and integration onto the DIM 


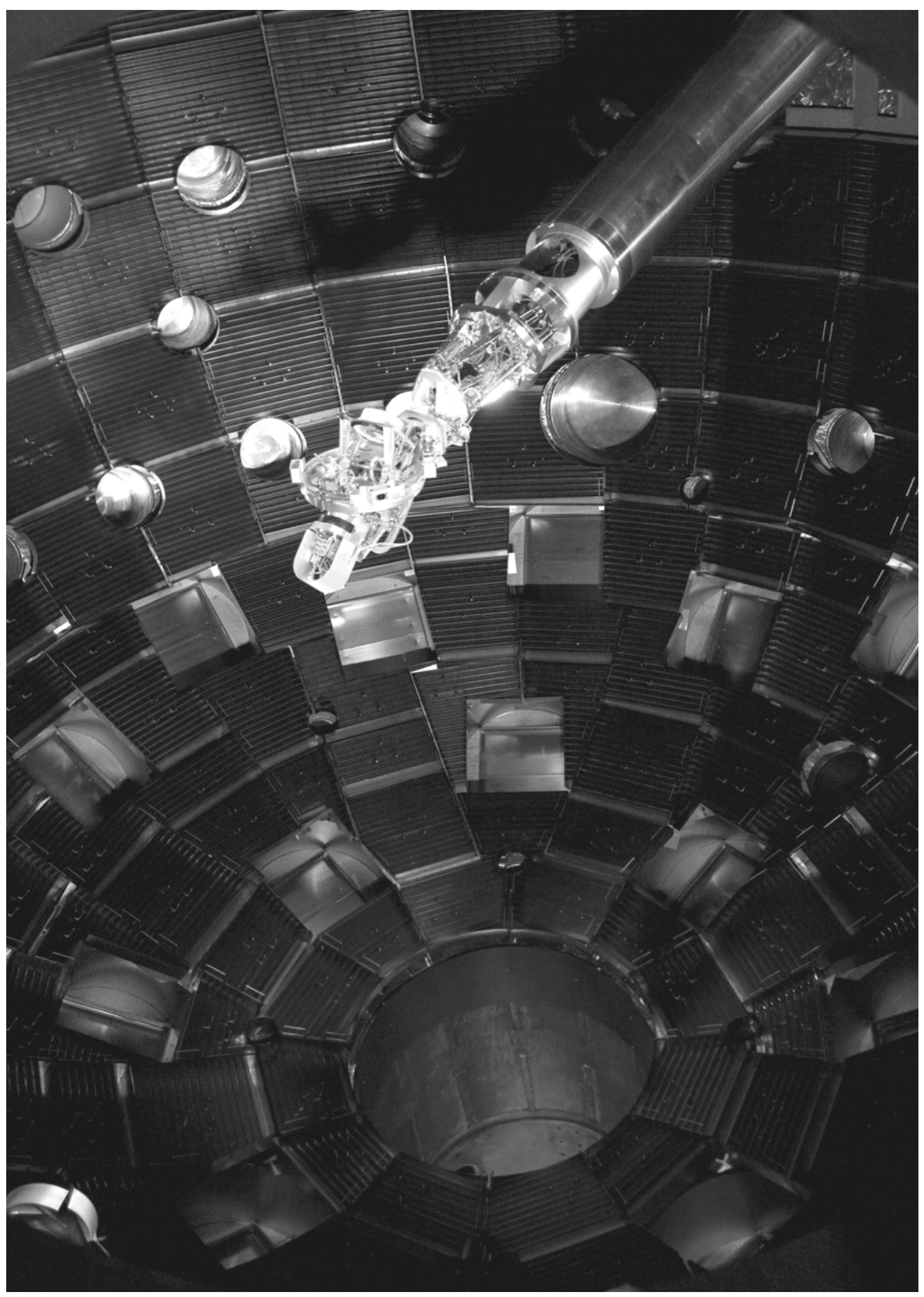

Figure 12 FODI instrument at the end of the DIM in the NIF target chamber 